The Geneva Papers on Risk and Insurance, 7 (No 25, October 1982), 279-289

\title{
The ASIR Model - An Introduction
}

\author{
by Lawrence Galitz *
}

This special edition of the Geneva Papers is devoted to our work on the ASIR model - work that has now extended over a four year period. This introduction sets out the background to the project, outlines some of the capabilities of the model and summarizes the main features of the ASIR system. Two papers report the results of research studies carried out at Bangor to investigate the effects on insurance companies of inflation, high interest rates and rapidly fluctuating exchange rates. Next, there is a contribution from a planning actuary from within the insurance industry, who has actually made use of the system in the course of his work. Finally, some sections from the 200 page user manual are reproduced here to give the reader an impression of how the model is used in practice.

The aim in preparing this edition has been to produce a balanced document that will explain what the ASIR model is, what it is capable of, how it may be used, and where ASIR can be applied.

\section{Evolution of the ASIR Model}

We start this introduction to ASIR right at the beginning in 1978, when the original idea for the project came about. We at Bangor were searching for a means by which people within the insurance industry and researchers could evaluate new ideas in insurance and attempt to shed light on some topical problems. The trouble with many of the conventional problem solving tools was that they were too slow, and by the time an insurance problem had been researched, analysed, and a solution proposed, the original problem had often gone away or been solved by some other means.

Our answer was to propose the construction of a simulation model, a test-bed for insurance that could be used to investigate the behaviour of an insurance company under a wide range of different circumstances. In just the same way as a model aeroplane in a wind tunnel tells the designers how the real plane would behave, so the insurance model would tell the user how the insurance company in real life would behave.

The proposal for an advanced insurance/ reinsurance simulation model was submitted to the Geneva Association in September 1978 and it was fortunate that U.K.

* Director of Studies, Department of Economics, University College of North Wales, 
they had the foresight to realise the potential that such a system would have. Funded by a grant from the Geneva Association, the project started at the Institute of European Finance, Bangor, in January 1979. Just a year later, the very first basic working version was demonstrated to the Association of Economists in Insurance in February 1980. More features were added, and the model was ready for its first presentation to the Board of the Geneva Association in July 1980.

One of the highlights in the history of the ASIR project was the January 1981 Corresponding Members meeting. At this event, fifty participants from thirteen countries were involved in a two day intensive ASIR Workshop. After a morning briefing session the participants, split into groups of four and five, were able to enter their data, perform simulations, and see the results. This cycle of activity was repeated many times, and was a valuable experience for all those concerned. From the participants' viewpoint, it enabled them to become familiar with the use and capabilities of ASIR ; from our viewpoint, it provided us with many useful comments and feedback on how we could improve on our work.

The suggestions we received led to a number of very helpful enhancements, and these were completed ready for the July 1981 presentation to the Board of the Geneva Association.

At about the same time, another important development started to take place. The computer service organisation TYMSHARE, who attempt to keep abreast of progress in the insurance world, first approached the Institute of European Finance and the Geneva Association with an interest in making ASIR more readily available to the insurance community. This led to an ASIR Workshop in November 1981, by which time there were several insurance companies making use of the ASIR system. The successful experience with that trial led to a formal ASIR launch, which took place in London in March 1982. ASIR was also featured as a technology exhibit at the World Insurance Congress in Philadelphia one month later.

Thanks to the feedback and suggestions we have received during the development of the project, ASIR has a much wider set of capabilities than was originally conceived ; for example, the model is some $60 \%$ larger than it was at its first unveiling. And, thanks to TYMSHARE, it can be readily accessed by any interested user almost anywhere in the world. The capabilities provided by the ASIR system are impressive, and in the next two sections we look at these in further depth.

\section{Concepts}

To understand how the concepts underlying ASIR were formulated, it is helpful at this point to refer to the objectives established at the outset of the project. These were twofold :

i) to create a powerful and flexible computer based modelling system to facilitate experimentation with insurance and reinsurance companies, and their interface with the operating environment ;

ii) to apply this system to the benefit of the insurance community. 
The first objective has already been satisfied now that the ASIR system is complete, and research studies using ASIR, like the ones reported later in this publication, illustrate how the second objective is being achieved.

The objectives embody some important keywords. Powerful and computer-based go hand-in-hand. It is necessary to have a system that is able to deal with the complexities and intricacies of insurance company operation, and only a computer-based model can provide analysis in sufficient depth in a reasonable time. Flexibility is also vital so that the diverse needs of different users can all be met.

Once these broad concepts were decided upon, more detailed concepts necessitated careful thought. A number of critical choices had to be made, and these are discussed in the following paragraphs.

\subsection{Type of model}

Here several choices were involved.

First, the selection between deterministic and stochastic models had to be made. This choice was not too difficult, because the essence of insurance is risk and uncertainty, and it was felt that any credible model must handle this explicitly, hence a stochastic model was necessary. However, it was recognised that some applications would be satisfied by a more simple deterministic approach. Bearing in mind the flexibility objective, it was decided to provide an option allowing the user to run ASIR in either stochastic or repeatable deterministic modes.

Next, the choice between descriptive and optimizing models had to be faced. Optimizing models are always attractive for the mathematician or operations research worker, but in this application it was felt that this kind of approach was not suited. There were several reasons for this. In the research application, the user usually wishes to carry out an investigative "what if ...?" study to see what would happen if an insurance company pursued a particular course of action in a specific environment. The aim is to describe rather than prescribe; hence a descriptive model is all that is necessary. In a practical corporate planning application, optimizing models for global corporate management are unable to evaluate all the quantitative and qualitative factors involved. A descriptive model is again better suited because it can present to top management the results of adopting a particular strategy, but leave the final decision to management on which course to take. Thus, ASIR is just a descriptive model.

Finally, there was the choice between accounting and empirical models. An accounting model contains straightforward and indisputable accounting logic, and nothing else. An empirical model also contains some behavioural equations governing such matters as market shares in a competitive pricing environment. It would have been attractive to build a model that would simulate, amongst other things, the number of policies that would result from a particular set of premium prices. However, building such a model is fraught with difficulties, not least of which is the problem of keeping the model up to date. Furthermore, most empirical models start with the assumption that relationships remain constant over time, an assumption that frequently does not hold in practice. To make ASIR more readily acceptable, it is purely an accounting 
model, but it has very comprehensive facilities enabling the user to enter data possessing any kind of empirical relationship. Furthermore, the system embodies accounting logic applicable to many countries, and the appropriate logic can be selected at will by the user.

\subsection{Implementation of the model}

Here as before, several decisions are involved.

From the user's viewpoint, there are two major methods of running a model : batch or interactively.

In the batch mode, the user prepares his data carefully, and then submits the data as a complete job to the computer system. The job is placed in a queue to await its turn with other jobs being scheduled. When the job is eventually run, it either succeeds or fails, and there is no recourse to the user in the latter case. Batch models can generally be larger in scope, and can exploit more of the computer's facilities, but there are often delays in obtaining results from batch models, which can stretch from hours to days.

Using the computer interactively is usually more efficient from the user's point of view, because he can converse directly with the computer. Working at a VDU or printing terminal, the user can enter data, execute simulations, and see the results, all within the space of a few minutes. Using the computer in this way enables the human and machine to work much more closely together, harnessing the best abilities of each. The human can exercise judgement, experience and hunch, while the computer can carry out the huge volume of calculations necessary both effortlessly and free of errors.

ASIR is flexible in this regard. All the processes involving contact with the user are completely interactive. This applies to the dedicated data entry system, the special data editing system, and the utility for listing information contained in any of the user's data files. The main processing programs can be run either interactively or in the batch, whichever suits the user at the time. If the user wishes to see the results straight away, or wants them printed at his terminal, the simulation can be carried out interactively on-line; the programs have been designed compactly enough to allow this. On the other hand, if the user wishes to submit a group of simulation runs to the computer, and does not want to wait around for the results to appear, they can be grouped together and submitted as a batch job. This is a further illustration of the concept of flexibility that has been an integral part of the design process.

\section{Capabilities}

In the broadcast terms, ASIR gives the user the ability to define an insurance company, a set of management strategies, and a background economic environment. Having done this, the user can investigate how the company would behave under these circumstances. With the flexibility that the ASIR system provides, the user at any time can change : 
- the nature of the insurance company under test ;

- the set of management strategies that the insurance company pursues ;

- the economic environment against which the company operates

to explore a wide range of scenarios quickly, accurately, and with comparatively little effort.

ASIR presents few limits to the complexity of problem that may be tackled. For example, ASIR can handle :

- up to to 9 active insurance or reinsurance companies ;

- up to 40 different kinds of risk portfolios ;

- up to 3 separate reinsurance treaties for each risk ;

- up to 9 different kinds of reinsurance treaty ;

- up to 20 countries and currencies ;

- up to 40 different investment portfolios ;

- a wide range of differing operating environments ;

- any length of simulation run ;

- 3 different modes of operation, including both deterministic and stochastic simulations ;

and even these limits can be extended if necessary.

To give a better idea of what these capabilities can mean for the user, the following paragraphs provide further explanation on each one.

Companies: While it is possible to set up a network of insurance companies linked by reinsurance treaties, most users wish to explore the operation of just a single company. This is possible through the existence within ASIR of a "shadow company" which can be the second party in any reinsurance contract. For the user simulating a direct company, the shadow company can be the reinsurer(s) who underwrite all reinsurance treaties. For the user who has set up just a single reinsurance company, the shadow company can act as the direct insurer(s) who originate the business. In this way, the user is spared the need to provide unnecessary information to the model.

Risks : ASIR defines two kinds of risk class. The GROUP risk describes the type of risk that comprises a portfolio of similar risks, for example, part of a motor portfolio. The VLR risk is a single high value risk, such as an oil platform. The user specifies the precise characteristics of each of the risk classes by entering a set of parameters. There is scope to specify virtually any kind of premium and claims experience, and the behaviour of each risk class simulated can be tailored to fit a very wide range of real life risks.

Reinsurance: For each of the risk classes simulated, the user can have up to three specific reinsurance treaties. Each of these can be selected from nine different kinds of treaty, covering all the common forms of proportional and non-proportional treaty. 
Countries and Currencies: With insurance being very much an international business, ASIR can deal with twenty currencies and countries of operation, so that the user can simulate premiums collected in one currency, claims settled in another currency, and reinsurance being arranged through a third country.

Investments : Each of the forty investment portfolios can comprise a mixture of three kinds of fixed rate investment, three kinds of equity, and investment in property. In eras of high interest rates, the return from the investment portfolio makes a significant contribution to insurance company performance. With ASIR, users can experiment with alternative investment strategies to evaluate their relative merits.

Operating Environment: Here, as with most other parts of the model, there is considerable flexibility afforded to the user. He can establish a wide set of economic backgrounds encompassing interest rates, inflation rates, economic growth, and market indices for property and equities. Furthermore, a separate set of information can be entered for every country of operation. With such a wide scope, the data entry requirements could be formidable, but ASIR features a special interpolator facility which means that the user need only enter the key turning points in the economic environment, and the model will fill in the rest of the detail.

Simulation time: The fundamental time unit within ASIR is one year, though this can be broken down into quarters in reports. A simulation can be as short as one year, or may extend longer. For short- and medium-term planning, a five or ten year simulation is not uncommon. ASIR can also be used for solvency studies testing insurance companies to the limits; here, simulations extending for thousands of years are quite feasible.

Reports : Some computer systems inundate the user with sheaves of printout, a large part of which can be irrelevant to the user's need. ASIR provides only as much detail as the user requires. At one extreme, the model allows the user to get an overview of the worldwide operations of an insurance company, or he can choose to concentrate just on one country, one branch, or even a single risk, in much greater detail. These results are usually presented in the form of clearly laid out numerical reports at various levels of aggregation, depending on the user's wishes. However, a facility added recently enables the user to collate and present information graphically in the form of multicoloured pictures.

Simulation modes: The simplest simulation mode is deterministic, where all simulated random events return their expected value (in the statistical sense). The next mode is the sensitivity analysis mode where random events do take place within the model, notably in the generation of claims, but the randomness is precisely repeatable from one simulation run to the next. This means that a user experimenting with changing other parameters, for example the operating environment or a management strategy, will know that the change in results is solely due to the change in his data and not because of changes in the occurrence of claims. The third mode is the fully stochastic mode, where ASIR can be run as a Monte-Carlo model, accumulating results over a large number of simulations.

The foregoing can really only give a surface impression of the full capabilities of the ASIR system, a fuller description being given in the 200 page Users Manual 
available from the Geneva Association. However, none of these capabilities would be of any use unless there were tangible benefits accruing from using the model. For research applications, the benefits are obvious, because ASIR provides a test-bed on which the researcher can experiment with the behaviour of an insurance company under a wide range of circumstances. Such a simulation model was not generally available before. For the commercial user, the results are also tangible, and are discussed now within the context of decision support systems.

\section{Decision support systems} layers :

The use of computers in business can, generally speaking, be split into three

i) at the bottom layer, the bulk of computer processing is carried out in the day to day running of an organisation; this routine data processing is of course essential for a company to perform ;

ii) at the next layer, computers are used to summarise the data processing carried out, and provide the management information systems necessary to make short term operational decisions ;

iii) at the top layer, computers can be used to carry out complex analyses of a company's present position, and projections of its likely future prospects under a number of possible scenarios.

As one moves through the stages, the volume of processing declines, but the value of the information produced increases. At the top layer, the kind of information obtainable from modelling systems such as ASIR can be invaluable in top level strategic decision making.

All the time, the management of insurance companies needs to make decisions. The correct decisions can only be taken by informed management, and management can only be informed if they have the right information. Typically, the information that management wants and needs is answers to "what if ...?" questions like :

- what if we delay raising our premiums by three months?

- what if we invest long but interest rates rise against our expectations?

- what if we suddenly have an adverse claims experience $25 \%$ worse than we expected?

These and many other "what if ...?" questions can be asked, and ASIR will provide the answers. Furthermore, in carrying out the simulations involved, ASIR will take into account all the delicate links and interrelationships that operate within an insurance company, and will cope with all the ramifications involved. An example would be the scenario where adverse claims led to a drain on liquidity, necessitating some investments to be realised at a loss, this further depressing profits, diminishing capital and possibly threatening solvency. ASIR can follow all the consequences through to their conclusion.

Thus, the major benefit in the corporate planning application is the provision of better information, because with better information, better decisions can be taken. 


\section{Using ASIR}

Right from the start it was intended that ASIR should be capable of being used by people who were not computer experts. For this reason, the ASIR system has been designed to be relatively easy to use, and with a few hours familiarisation, use of the model can be mastered even by people who have never used a computer before.

There is a typical "cycle-of-use" that has four steps :

i) the user enters a new set of data which is stored in computer files in the user's own library area;

ii) simulations are carried out using the data previously entered;

iii) the user analyses the results; this suggests new alternatives to be evaluated;

iv) the user makes changes to previously entered data and returns to step (ii).

As an example, the user may enter his original set of data describing the most likely forecast economic background, and carry out the first simulation. He may then wish to edit the economic environment data to investigate the effect on the insurance company of better or worse circumstances. These steps can be executed as many times as the user wishes until he is satisfied that he has all the results he wants.

The internal structure of the ASIR model is specifically designed to reflect these steps. The IMIDES data entry system provides a fully prompted conversational system to guide the user through the task of entering a new set of data from the user, checking it for consistency (step (i)). The ASIRRG and ASIR systems are the main simulation programs that respectively permit summary and detailed simulations to be carried out (step (ii) ). The results produced by the main simulation programs appear automatically, but there is also a LISTER utility to provide print-outs of the sets of previously entered data currently stored in the users' library area. Finally, the interactive EDITOR system permits previously stored data to be inspected and edited ready for new simulations (step (iv)).

A more detailed description of using ASIR is given in chapters fifteen and sixteen of the Users Manual, reproduced later on in this edition.

\section{Applications}

ASIR can be applied to most tasks where a user wishes to study the behaviour of an insurance company under a range of possible economic environments or management strategies. There are several areas where obvious applications arise, and these fall naturally under four main headings.

\subsection{Short term planning}

Most companies undertake a budgeting exercise at some stage through the year to set targets and to manage the application and use of resources. In the past, this was predominantly a manual exercise taking weeks or even months to carry out. Owing to the amount of effort involved, there was usually only time to evaluate one alternative, 
which became the plan. In some complex areas, it was not always possible to follow through with all the ramifications of a particular management decision.

The short term planning application is probably the classic application where models can best be put to use, and ASIR is no exception. The corporate planner in an insurance company is usually faced with the problem that, while he knows his company's present position, he does not know how it will be affected by future economic circumstances or proposed management actions. With ASIR, the planner can set up a fairly accurate representation of his company, or just a part of it, and experiment with its behaviour. The results that ASIR will produce will define the effects of the alternatives being explored, and the way that ASIR behaves echoes the way that the company in real life would behave under those same circumstances. With the "cycle-of-use" described earlier, the planner can explore a wide range of possibilities quickly, accurately, efficiently, and without great cost. In this way, the uncertainty surrounding management decisions can largely be replaced by foresight.

\subsection{Long range planning}

Planning at the strategic end of the time scale can often be a more difficult task because of the remoteness of the events that have to be considered, and the greater uncertainty associated with them. ASIR helps in this application because it makes no difference to the operation of the model whether it is dealing with timespans of twenty to fifty years, or two to five years.

When ASIR is used for simulations covering lengthy periods, the user typically sets up a somewhat more simplified model because it is easier to see the important behavioural changes when the results are not obscured by the intricate detail found in short term planning exercises. Using ASIR here can be particularly advantageous, because the model can project company performance for decades into the future. ASIR can thus reveal the potential build up of adverse circumstances that would not be apparent from shorter simulations, and which would not manifest themselves in real life for many years. Having such a far-sighted look into the future gives the company ample time in which to effect suitable alterations in its strategy. are :

Examples of the "what if ...?" questions that can be asked in this application

- what are the financial prospects associated with entering a new line of underwriting business?

- what are our likely capital needs in the future?

- are our reinsurance arrangements fair? are they optimum ?

\subsection{Education and training}

In a world of even increasing complexity and change it is more than ever necessary to provide effective education and training. One of the best methods of learning, where it can be applied, is learning by doing. A student will be confronted by a problem, 
and must find his own solution; as he progresses he will learn by his experience and his mistakes.

Those involved in the management of insurance companies must have a detailed understanding of how the whole company operates. This can be gained over a period of time as experience develops, but it would be helpful in many applications if a more intensive learning experience could be arranged. This is especially relevant in higher education centres dealing with insurance and insurance company management. Clearly there could be disastrous consequences if students were let loose on a real insurance company to see how it behaved under various circumstances. Besides, in real life years have to pass before the full effects of their decisions would be perceived.

A reasonable alternative is to use the ASIR model as a substitute for the real company. The learning process would take place as a series of steps :

i) The tutor would set up a representation of the company on the model, and present the student with a description of the insurance company's current position. Either general situations would be set up in order to teach the general principles of insurance company operations, or specific circumstances would be simulated in order to give detailed insight into a particular problem.

ii) The student would evaluate this information to see where the insurance company is now, and where it is likely to be in the future, identifying any problem areas in the process.

iii) The student would then devise strategies that were likely to surmount the problems. These would be supplied to the ASIR model, which would process them and produce the resulting financial and operating statements.

v) The student would see how well his proposed strategies worked in practice, and would revise them accordingly.

Steps (iii) and (iv) would be repeated until the situation established by the tutor had been explored completely. Further scenarios could then be tried.

By using this technique, the student's understanding and ability to make the correct decisions will improve each time the loop is traversed, and valuable experience is gained all the time. The positive feedback showing the results of the student's decisions immediately they are made is also extremely effective.

\subsection{Research}

As will be recalled from the first section, the purpose for which the ASIR model was built initially was research. The system provides the means by which individuals undertaking research into insurance related problems can test their hypotheses on a realistic simulation of an insurance company. In this regard, ASIR provides a flexible range of options that were previously unavailable. Already, two research projects based on the ASIR model have been completed, and are reported later on in this edition. It is hoped that more will follow, so that others may take advantage of the unique facilities that the ASIR model provides. 


\section{Summary}

The intention of this introduction is to provide the reader with an impression of what the ASIR model is, what it is capable of, and where it may be applied. The remaining articles in this special edition of the Geneva Papers go further. The two research reports provide a more tangible illustration of the kind of results obtainable through use of the ASIR model, while the contribution from a planning actuary gives one view on the potentials of ASIR within the insurance industry itself. Finally, the extracts from the Users Manual give a clearer impression of the use of the model in practice. We hope they will provide sufficient stimulus to encourage some readers to make use of the ASIR model in their own work.

\section{REFERENCE}

BROWN, M., and GALITZ, L.: "The ASIR System - Users Manual ", Etudes et Dossiers of the Geneva Association, 52, November 1981. 